\title{
Investigation of the chiroptical behavior of optically active polyaniline synthesized from naturally occurring amino acids
}

\author{
Sudha ${ }^{1}$, Devendra Kumar ${ }^{1}$ and Mitsumasa Iwamoto ${ }^{2}$ \\ The chiroptical properties of optically active polyaniline PANI(+)-HCSA emeraldine salt products in dimethylformamide (DMF) \\ depend strongly on stereochemistry and steric crowding due to the presence of different moieties on the amino acids. Amino \\ acids including L-arginine, L-proline, L-leucine, L-isoleucine, L-phenylalanine and L-lysine hydrochloride were used as precursors \\ in emeraldine base/DMF solutions prior to acid doping with (S)-(+)-10-camphorsulfonic acid. Characterization of these \\ materials was carried out using CD, UV-Vis and FTIR spectroscopic techniques. Our results indicate that the optical activity can \\ be maintained for longer times in the solid and solution phases owing to the interactions and stabilization of polyaniline by \\ hydrogen bond formation between the amino-acid molecules and the amine $(\mathrm{NH})$ and imine $(-N=)$ sites on the emeraldine \\ base form, while preserving the stereo-selectivity of the polymer chain. This paper addresses the factors responsible for \\ influencing the chiroptical properties in helical conducting polyaniline and the chiral discrimination of different amino acids. \\ Polymer Journal (2013) 45, 160-165; doi:10.1038/pj.2012.127; published online 20 June 2012
}

Keywords: circular dichroism; helical conducting polymer; hydrogen bonding; optical activity; retention of configuration

\section{INTRODUCTION}

A new class of polymers, known as electroactive polymers or intrinsically conducting polymers, characterized by extended $\pi$-conjugation along the polymer backbone, has emerged as a group of advanced materials owing to their characteristics and potential applications in various devices. These conducting polymers are of great scientific and technological importance because of their electrical, electronic, magnetic and optical properties. ${ }^{1}$ Electroactive and inherently conducting polymers such as polypyrrole, polythiophene, and polyaniline (PANI), among others, are organic semiconductors. When helicity is induced in these conducting polymers, they are of great use in circularly polarized electroluminescence devices, ${ }^{2,3}$ chirality-modified electrodes, ${ }^{4}$ stereo-selective analysis for the selective transport of enantiomers, ${ }^{5}$ electrochemical switches, surface-modified electrodes, chiral chromatography, membrane separation technologies, sensors based on the combination of conductivity, chiral selectivity and molecular recognition, electrodes for enantioselective recognition or one that are capable of performing bio-electro-synthesis, light-emitting diodes, and electrode materials in field-effect transistors. ${ }^{6,7}$ Thus, helical or optically active conducting polymers are currently attracting the attention of researchers. ${ }^{8}$ Optically active polyaniline emeraldine salts can be prepared either by electrochemical polymerization ${ }^{9,10}$ or by the doping of emeraldine base (EB) in organic solvents with chiral agents. ${ }^{11,12}$ Subsequently, chiral anions are incorporated onto the main chain of PANI as counter-ionic dopants through electrostatic interaction or hydrogen bonding. Hence, the optical activity can be preferentially observed owing to the adoption of one-handed helical conformations of polyaniline. It has been found that the chiroptical properties of doped polyaniline depend on the nature of the dopant, the reaction medium, the water content and the order of mixing of the reagents during doping. ${ }^{13-16}$

In this paper, we report on discriminations and factors associated with amino acids that can affect the chiroptical properties of PANI ++ -HCSA emeraldine salt products prepared by doping of PANI with $(+)$ HCSA in dimethylformamide (DMF) solvent.

\section{EXPERIMENTAL PROCEDURE}

\section{Materials}

All amino acids, including L-arginine (L-Arg), L-proline (L-Pro), L-leucine (LLeu), L-isoleucine (L-Ile), L-phenylalanine (L-Phe) and L-lysine (L-Lys) hydrochloride, were purchased from Spectrochem Pvt. Ltd. (Mumbai, India), and $\mathrm{S}(+)$-camphorsulfonic acid (HCSA) was purchased from Himedia Laboratories Pvt. Ltd. (Mumbai, India). Guanidine hydrochloride was procured from Sigma-Aldrich (St Louis, MO, USA). Aniline and $\mathrm{HCl}$ were supplied by Chemical Drug House Pvt. Ltd. (Mumbai, India), whereas ammonium persulphate was purchased from Merck Specialties Pvt. Ltd. (Mumbai, India)

${ }^{1}$ Department of Applied Chemistry and Polymer Technology, Delhi Technological University (Formerly Delhi College of Engineering), Delhi, India and ${ }^{2}$ Department of Physical Electronics, Tokyo Institute of Technology, Tokyo, Japan

Correspondence: Professor D Kumar, Department of Applied Chemistry and Polymer Technology, Delhi Technological University (Formerly Delhi College of Engineering), Shahbad Daulatpur, Main Bawana Road, Delhi 110042, India.

E-mail: drdkumar@yahoo.co.uk

Received 31 December 2011; revised 27 April 2012; accepted 1 May 2012; published online 20 June 2012 
DMF was procured from Qualigen Fine Chemicals (Mumbai, India). The aniline was double distilled and stored at low temperature prior to use. Other chemicals were used as received without further purification. Double-distilled and demineralized water from Millipore (India) Pvt. Ltd. (Bangalore, India) was used throughout the studies.

\section{Synthesis and doping of polyaniline EB}

Supplementary Figure S1 shows a schematic illustration of the procedure followed for the synthesis of polyaniline (EB) and doping of polyaniline (EB) by chiral amino acids.

Synthesis of polyaniline. Chemical polymerization of aniline was performed with the aid of ultrasonic irradiation using an Ultrasonicator (Model USB2.25) from Accumax India (Delhi, India). A $0.2 \mathrm{M}$ solution of freshly distilled aniline was prepared in a $1 \mathrm{M} \mathrm{HCl}$ solution and cooled to $0-5^{\circ} \mathrm{C}$. An aqueous solution of equimolar ammonium persulphate $(0.2 \mathrm{M})$ was added drop wise $(2.5 \mu \mathrm{l}$ in one lot) under sonication for $30 \mathrm{~min}$ while maintaining the temperature of the reaction mixture below $5{ }^{\circ} \mathrm{C}$. A dark green precipitate was obtained and filtrated, and it was then washed with a $1 \mathrm{M} \mathrm{HCl}$ solution several times, followed by washing with deionized water. It was then finally dried and powdered. The final product was dedoped by a 1:1 aqueous ammonia solution to obtain the EB form of polyaniline.

Doping of the EB. A stock solution of the EB form of polyaniline was prepared by dissolving $3.8 \mathrm{mg}$ of polymer in $5 \mathrm{ml}$ of DMF solvent. Each of the above-listed amino acids were added to the EB solution separately at concentrations of $30 \mathrm{~mm}, 60 \mathrm{~mm}, 90 \mathrm{~mm}$ and $120 \mathrm{~mm}$ and stirred for $1 \mathrm{~h}$. Each solution was divided into two parts: (1) the spectroscopic studies were carried out within $30 \mathrm{~min}$ of chiral acid doping (shown as L-Arg $30 \mathrm{~mm}$ ) and (2) the studies were carried out after $24 \mathrm{~h}$ of chiral acid doping, in which cases the samples are represented by the addition of a prime to the data (shown as L-Arg' $30 \mathrm{~mm}$ ). After addition of the chiral acid, that is, (+ )-HCSA, polyaniline was converted into the PANI ++ )-HCSA.ES product. A similar procedure was followed with guanidine, which is an achiral amino acid.

Spectroscopic studies. UV-Visible spectra were recorded using a double-beam UV-Vis spectrophotometer (Model UV5704SS) ECIL, Hyderabad, India. These spectra were taken at dilution factor of 3:7 (volume by volume) of the final PANI $(+)$-HCSA.ES product in DMF. The corresponding circular dichroism spectra were recorded using a chira-scan circular dichroism spectrometer (Applied Photophysics Ltd, Surrey, UK) at a $50 \mathrm{kHz}$ frequency and wavelength step size of $1 \mathrm{~nm}$. The UV-Visible and CD spectra were recorded with a $1-\mathrm{cm}$ path-length. The CD and UV-Visible spectra of the chiral and achiral aciddoped solutions were taken in sequence with increasing concentration of the respective amino acids within $30 \mathrm{~min}$ of preparation and after $24 \mathrm{~h}$ of preparation. The Fourier transform infrared (FTIR) spectra of PANI and $(+)$-HCSA-doped PANI were taken on an FTIR spectrophotometer (Model 2000, Perkin Elmer, Waltham, MA, USA).

\section{RESULTS AND DISCUSSION}

Figure 1 shows the chemical structures of the amino acids used as precursors for induction of helicity in the polymer. These structures are helpful for understanding the changes in the chiroptical properties of optically active polyaniline in comparison to its parent polymer, polyaniline. In this, we are mainly reporting on spectroscopic studies such as UV-visible, FTIR and CD data to understand the chiroptical behavior of optically active polyaniline.

\section{UV-Visible spectra}

Figure 2 shows the UV-Visible spectra of the EB form of the polyaniline stock solution in DMF with a characteristic excitation bands at $621 \mathrm{~nm}$ and a $\pi-\pi^{*}$ band at $334 \mathrm{~nm}$. With the addition of $0.1 \mathrm{~m}(+)$-HCSA to the solution, the color of the solution changed from blue to green. In this case, absorption bands were observed at 344,417 and $861 \mathrm{~nm}$, depicting a $\pi-\pi^{\star}$ band, and two polaron bands<smiles>N=C(N)NCCC[C@H](N)C(=O)O</smiles><smiles>CC[C@H](C)[C@@H](N)C(=O)O</smiles>
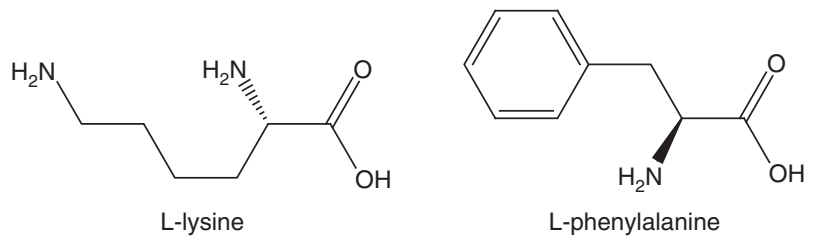

L-phenylalanine

Figure 1 Chemical structures of amino acids.

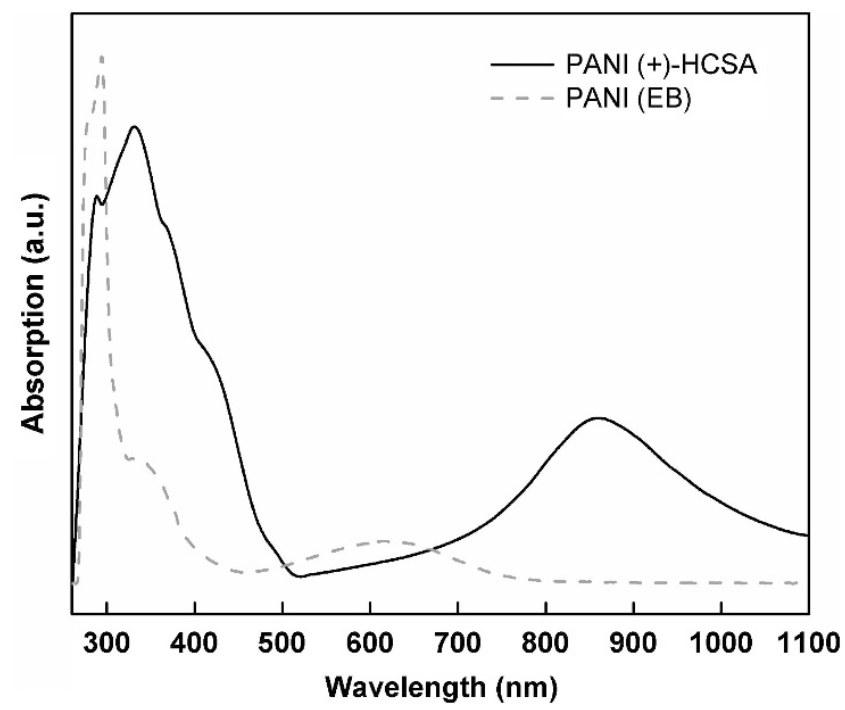

Figure 2 UV-Vis spectra of PANI (EB) and PANI + +)-HCSA in DMF. A full color version of this figure is available at Polymer Journal online.

were observed with a 'compact coil' conformation that agrees well with the literature. ${ }^{15}$ The position and intensity of the UV-Visible absorption bands of PANI $(+)$-HCSA can be related to the conformation and conjugation length of the polymer. The localized polaron band in the range of $750-850 \mathrm{~nm}$ is considered as characteristics of the 'compact coil' conformation of the PANI chains.

The UV-Visible spectra of the EB form of the polyaniline and PANI $(+)$-HCSA.ES product solutions containing L-Arg show that, as the time period and concentration of amino acids increase, the emeraldine salt form changes to the EB form. It is also clear from the UV-Visible data that there is a band shift from higher to lower wavelength as shown in Figure 3. It is also reported that the PANI (+ )-HCSA.ES solution containing $30 \mathrm{~mm}$ of L-Arg remained stable even after 15 days. 


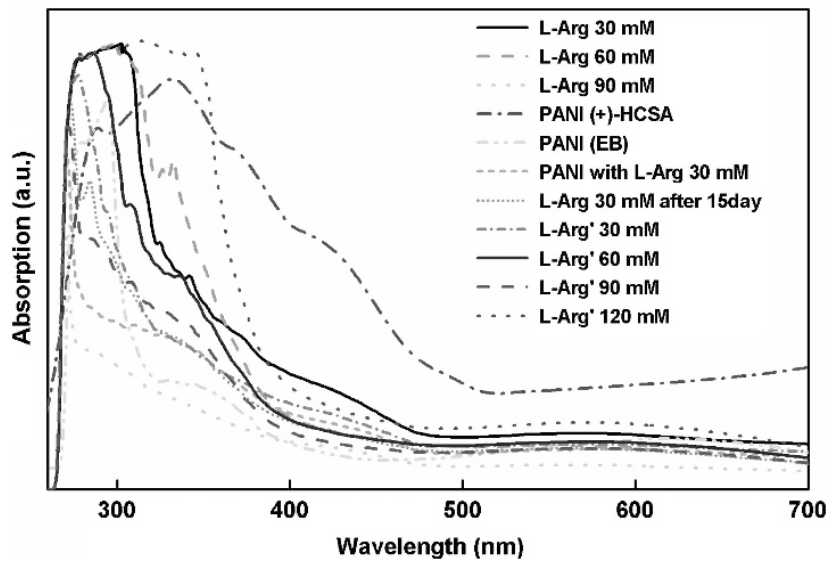

Figure 3 UV-Visible spectra of PANI(+)-HCSA in 30-120 mm of L-Arg along with enantiomeric PANI(+)-HCSA and PANI (EB) in DMF. (L-Arg spectra were taken within $30 \mathrm{~min}$ of doping, while L-Arg' were taken after $24 \mathrm{~h}$ of doping). A full color version of this figure is available at Polymer Journal online.

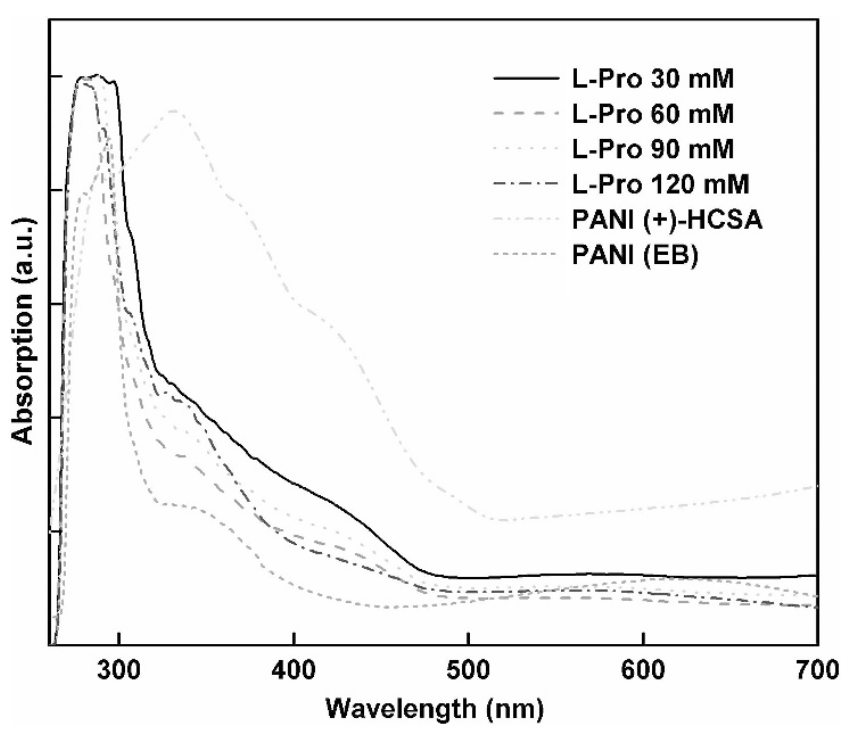

Figure 4 UV-Visible spectra of PANI(+)-HCSA in 30-120 mm of L-Pro along with enantiomeric PANI(+)-HCSA and PANI (EB) in DMF. A full color version of this figure is available at Polymer Journal online.

In Figure 4, the UV-Visible spectra of the PANI(+)-HCSA.ES solutions containing L-Pro at concentrations higher than $90 \mathrm{~mm}$ show similar spectra to those of the EB. In the case of L-Leu, the UV-Visible spectra observed in the presence of $\geqslant 90 \mathrm{mM}$ of L-Leu is found to be similar to that of EB. In case of L-Ile, the characteristic bands of ES remain unchanged even at higher concentrations, but for the case of L-Phe, the characteristic bands of ES change to EB at only $90 \mathrm{~mm}$ of L-Phe. With L-Lys hydrochloride, the UV-Visible spectra corresponding to EB are obtained at concentrations higher than $30 \mathrm{~mm}$ of L-Lys. Supplementary Figure S3 shows a comparison between the UV-visible spectra of PANI (EB) and PANI (+ )-HCSA with $30 \mathrm{~mm}$ of chiral and achiral amino acids. These spectra reveal that achiral acids such as guanidine are not helpful for secondary doping, and this spectrum is entirely different from the spectra recorded for chiral acid doping.

\section{FTIR studies}

PANI and PANI $(+)$-HCSA.ES samples were also characterized by FTIR. The FTIR spectra of the EB PANI and PANI(+)-HCSA.ES

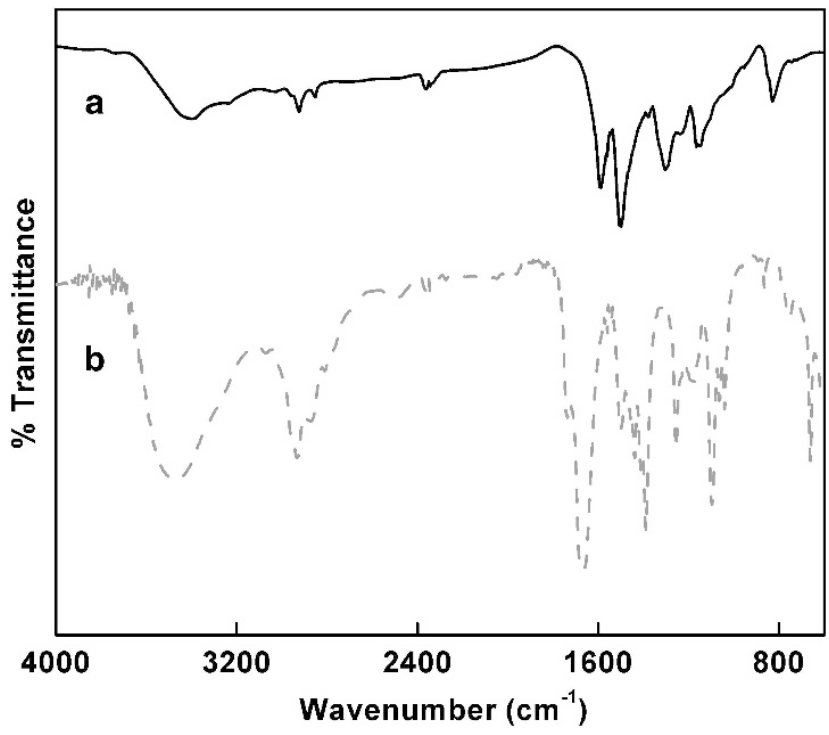

Figure 5 FTIR spectra of (a) PANI (EB) and (b) PANI(+)-HCSA containing $30 \mathrm{~mm}$ L-Pro. A full color version of this figure is available at Polymer Journal online.

products containing amino acids as precursors were recorded using $\mathrm{KBr}$-pressed pellets and Nujol medium. As the PANI(+ )-HCSA.ES products were prepared in DMF solvent and are reactive in nature, so nujol provides a protective coating, preventing sample decomposition during acquisition of the IR spectrum. Figures $5 \mathrm{a}$ and $\mathrm{b}$ show the characteristic peaks observed at 3435, 2923 and $1297 \mathrm{~cm}^{-1}$, which are attributed to the $-\mathrm{NH}$ stretching, $\mathrm{C}-\mathrm{H}$ stretching on an aromatic ring and C-N stretching modes, respectively, whereas peaks at 1484 and $1564 \mathrm{~cm}^{-1}$ show the presence of benzenoid and quinoid structures, respectively. The characteristic peak at $1121 \mathrm{~cm}^{-1}$ is due to aromatic amine stretching. The peak at $878 \mathrm{~cm}^{-1}$ is attributed to the out-ofplane hydrogen deformation of the aromatic rings in the PANI unit sequences. In Figure 5b, the characteristic peaks of $(+)$-HCSA appear at 1397 and $669 \mathrm{~cm}^{-1}$, which were found to be in close agreement with data reported in the literature. ${ }^{17}$ The characteristic peaks at 1667 and $1033 \mathrm{~cm}^{-1}$ are attributed to the $\mathrm{N}-\mathrm{H}$ bending and $\mathrm{C}-\mathrm{N}$ stretching modes in the amines, respectively, which are due to the presence of peptide bonds of the amino acids resulting in hydrogen bonding between amino-acid molecules and amine $(\mathrm{NH})$ and imine $(-N=)$ sites on the PANI chains.

\section{Circular dichroism studies}

Supplementary Figure S2 shows the circular dichroism spectra of PANI- $(-)$ HCSA and PANI- $(+)$ HCSA confirming the optical activity in opposite phases. In-situ developed $\operatorname{PANI}(+)-\mathrm{HCSA} /(-)$ HCSA solutions possess characteristic bands at approximately 295 and $445 \mathrm{~nm}$. The CD band at $295 \mathrm{~nm}$ is attributed to HCSA incorporation in the polymer, whereas the $445 \mathrm{~nm}$ band is attributed to the optical activity induced in the polymer backbone, as HCSA does not show any $\mathrm{CD}$ band in the visible region. ${ }^{18}$ The opposite direction of the band at $295 \mathrm{~nm}$ confirms the enantioselective incorporation of the HCSA, whereas the handedness of the polymer is visible from the band observed at $445 \mathrm{~nm}$.

The CD spectra of the PANI(+)-HCSA.ES products with and without amino acids have been reported to establish the helical or optically active nature of the samples. CD data measurements were carried out by adding different amounts of amino acids to PANI solution in DMF prior to doping with $0.1 \mathrm{M}(+)$-HCSA. It can be 


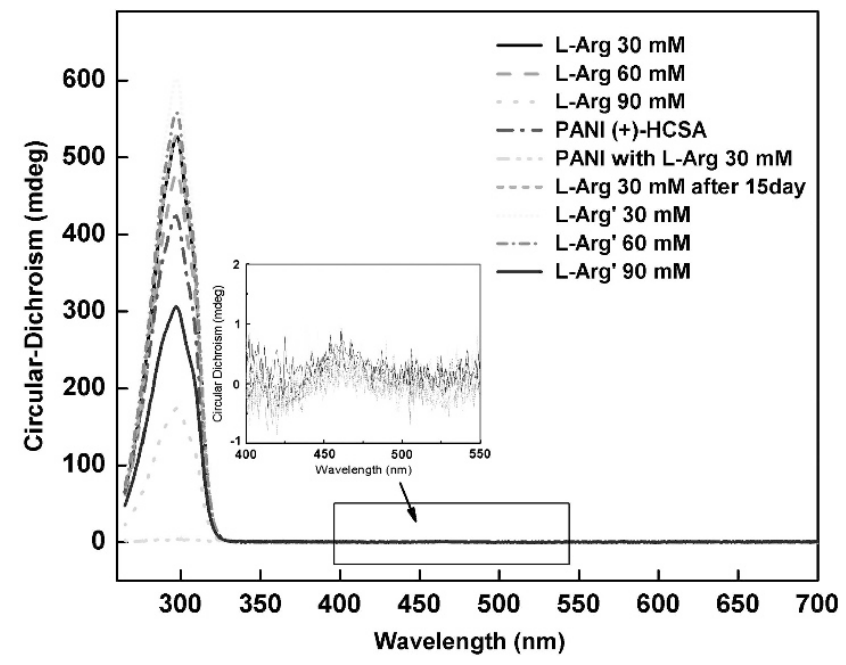

Figure 6 CD spectra of PANI(+)-HCSA in 30-120 mm of L-Arg along with enantiomeric PANI(+)-HCSA in DMF. (L-Arg spectra were taken within 30 min of doping, while L-Arg' were taken after $24 \mathrm{~h}$ of doping). A full color version of this figure is available at Polymer Journal online.

observed in Supplementary Figures S4 and S5 that only the chiral amino acids affect the helicity/optical activity of PANI $(+)$-HCSA.ES, whereas achiral amino acids such as guanidine do not affect the optical activity of PANI $(+)$-HCSA.ES. This difference is due to the importance of both electrostatic forces and hydrogen bonding, which are essential for helicity. However, in the case of guanidine, only hydrogen bonding is possible. Helicity has been induced by $(+)$ HCSA but the study of the effect of amino acids on these helical polymers is of prime importance.

It can be seen in Figure 6 that the CD spectra of PANI and PANI(+ )-HCSA solutions formed within $30 \mathrm{~min}$ in the presence of $\mathrm{L}$-Arg were different from those recorded after $24 \mathrm{~h}\left(\mathrm{~L}-\mathrm{Arg}^{\prime}\right)$ and 15 days. A remarkable decrease in the ellipticity with increasing time period was observed. This decrease indicates that an increase in the de-doping of ES to EB and diminishing hydrogen bonding between the amino-acid molecules and amine $(\mathrm{NH})$ and imine $(-N=)$ sites on the EB chains are responsible for such a remarkable change. The presence of 30-60 mM L-Arg had a significant effect on the intensity of the CD band of PANI $(+)$-HCSA.ES, whereas the presence of higher concentrations such as $90 \mathrm{~mm}$ caused a reduction in the intensity of the $\mathrm{CD}$ band because of an increase in $\mathrm{pH}$ of the reaction medium. The $\mathrm{pH}$ influences the extent of ellipticity and hydrogen bonding in the polymer during polymerization. In the case of $90 \mathrm{~mm}$ of L-Arg, the $\mathrm{pH}$ became alkaline: consequently, the extent of hydrogen bonding and further attack of chiral acids on the reacting sites decreased, which supports the remarkable decrease in the intensity of the CD band.

Figure 7 shows the CD spectra of the PANI $(+)$-HCSA.ES product solution in the presence of L-Leu taken within $30 \mathrm{~min}$ and the CD spectra observed after $24 \mathrm{~h}$. The CD band intensity of the doped solutions formed within 30 min was greater than the doped solutions formed after $24 \mathrm{~h}$. This result suggests that, as time increases, the intensity decreases owing to an increase in EB formation and a decrease in the extent of hydrogen bonding. Thus, the trend in the band intensity of L-Leu' $30 \mathrm{~mm}>\mathrm{L}-\mathrm{Leu}^{\prime} 90 \mathrm{~mm}>\mathrm{L}-\mathrm{Leu}^{\prime} \quad 60 \mathrm{~mm}$ $>_{\text {L-Leu }} 120 \mathrm{~mm}>_{\text {L-Leu }} 120 \mathrm{~mm}>_{\text {L-Leu }} 60 \mathrm{~mm}>_{\text {L-Leu }} 90 \mathrm{~mm}>$ L-Leu $30 \mathrm{~mm}>\mathrm{PANI}(+)$-HCSA was observed. This trend is due to an increase in the concentration of amino acids that contains only

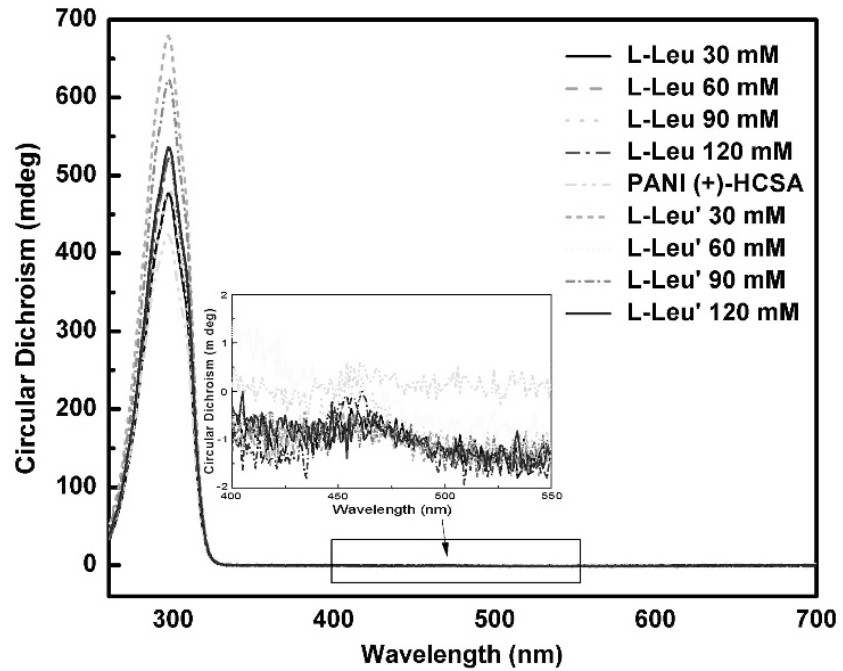

Figure $7 \mathrm{CD}$ spectra of PANI(+)-HCSA in 30-120 mm of L-Leu along with enantiomeric PANI + )-HCSA in DMF. (L-Leu spectra were taken within 30 min of doping, while L-Leu' were taken after $24 \mathrm{~h}$ of doping). A full color version of this figure is available at Polymer Journal online.

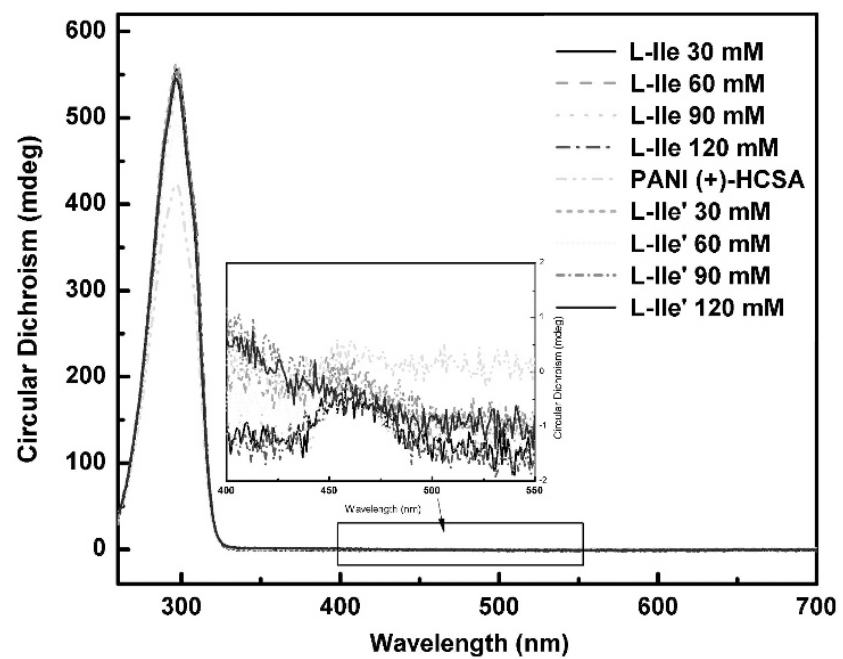

Figure 8 CD spectra of PANI(+)-HCSA in $30-120 \mathrm{~mm}$ of L-lle along with enantiomeric PANI(+)-HCSA in DMF. (L-Ile spectra were taken within 30 min of doping, while L-Ile' were taken after $24 \mathrm{~h}$ of doping). A full color version of this figure is available at Polymer Journal online.

alkyl groups. Thus, there is a remarkable decrease in the formation of EB with increasing time.

Figure 8 shows that the extent of the $\mathrm{CD}$ band intensities was not very distinct at different concentrations because they seem to be highly intense relative to the PANI $(+)$-HCSA solution. This result may be due to the presence of amino and methyl groups in opposite planes, causing weak interactions between the amino acids and PANI. The methyl group lies in the same plane in which the interactions occur; consequently, the steric effect reduces the extent of hydrogen bonding, whereas in the case of L-Leu, the methyl group is present in the opposite plane and a remarkable difference in intensity is observed.

In the case of L-Phe, the $\mathrm{CD}$ band intensity order due to variations in concentration is L-Phe $120 \mathrm{~mm}>\mathrm{L}-\mathrm{Phe}^{\prime} 30 \mathrm{~mm}>\mathrm{L}-\mathrm{Phe}$ $30 \mathrm{~mm}>$ L-Phe $\mathrm{C}^{\prime} 60 \mathrm{~mm}>_{\mathrm{L}-\mathrm{Phe}} 60 \mathrm{~mm}>_{\mathrm{L}-\mathrm{Phe}} 90 \mathrm{~mm}>\mathrm{PANI}(+)$ HCSA $>$ L-Phe' $90 \mathrm{~mm}$. The band intensity of the doped solutions 


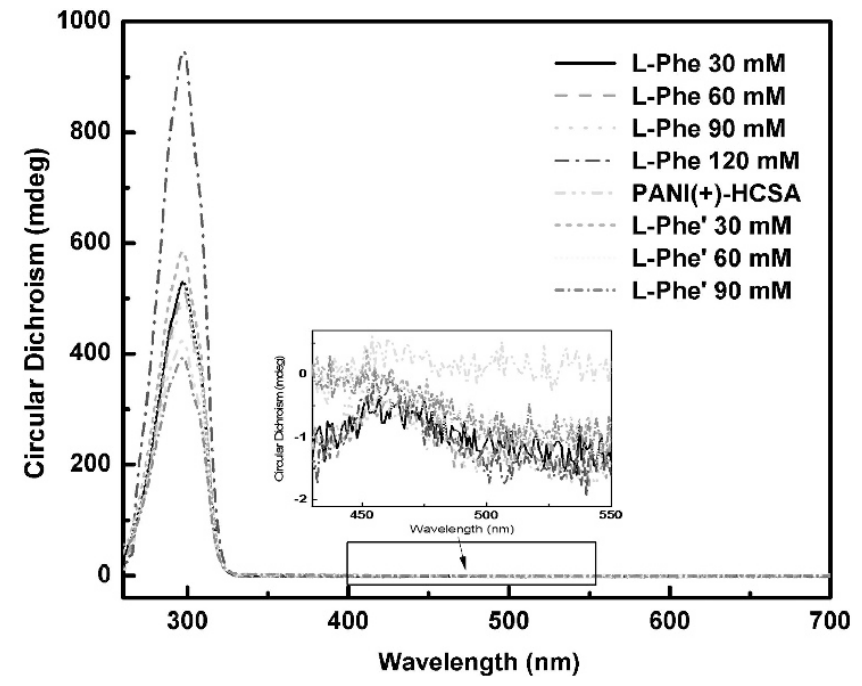

Figure $9 \mathrm{CD}$ spectra of PANI(+)-HCSA in 30-120 mm of L-Phe along with enantiomeric PANI(+)-HCSA in DMF. (L-Phe spectra were taken within 30 min of doping, while L-Phe' were taken after $24 \mathrm{~h}$ of doping). A full color version of this figure is available at Polymer Journal online.

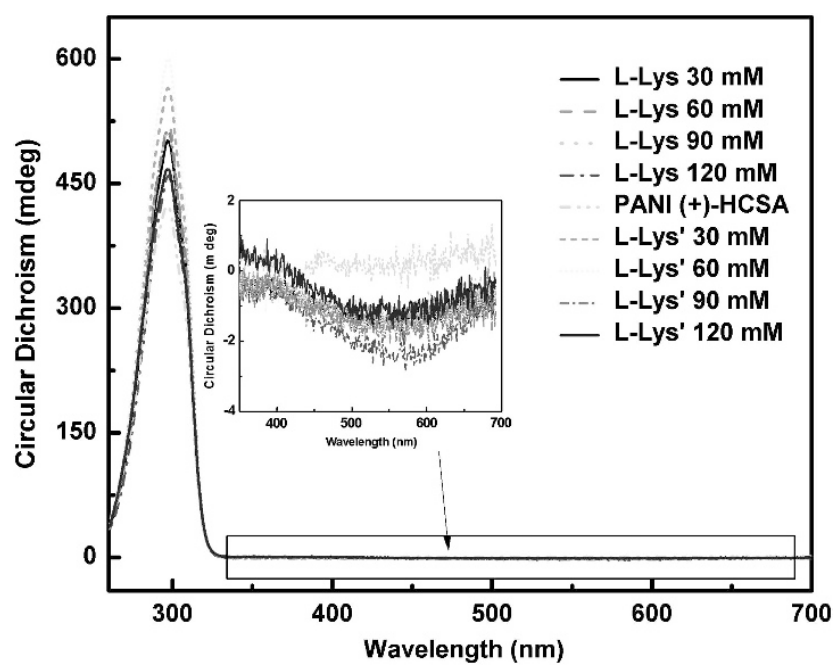

Figure $10 \mathrm{CD}$ spectra of PANI(+)-HCSA in 30-120 mm of L-Lys hydrochloride along with enantiomeric PANI(+)-HCSA in DMF (L-Lys spectra were taken within $30 \mathrm{~min}$ of doping, while L-Lys' were taken after $24 \mathrm{~h}$ of doping). A full color version of this figure is available at Polymer Journal online.

recorded within $30 \mathrm{~min}$ was found to be greater than that of the doped solutions recorded after $24 \mathrm{~h}$. Moreover, with an increase in the concentration of amino acids, the intensity generally decreases. In case of L-Phe' $120 \mathrm{~mm}$, the band intensity was observed to be abruptly higher than that of other samples as shown in Figure 9.

In the case of L-Lys, the $\mathrm{CD}$ band intensities were not easily distinguishable as they were more intense than that of the PANI $(+)$ HCSA solution as shown in Figure 10. It can be seen from the spectra recorded within $30 \mathrm{~min}$ that as the concentration increased, the band intensity decreased. However, this is not the case for solutions kept for longer periods of time. In the case of L-Pro, the CD band intensities observed within $30 \mathrm{~min}$ had higher band intensities than the PANI $(+)$-HCSA solution, as shown in Figure 11. When the spectra were taken after $24 \mathrm{~h}$, the band intensity of the $30 \mathrm{~mm}$ solution was

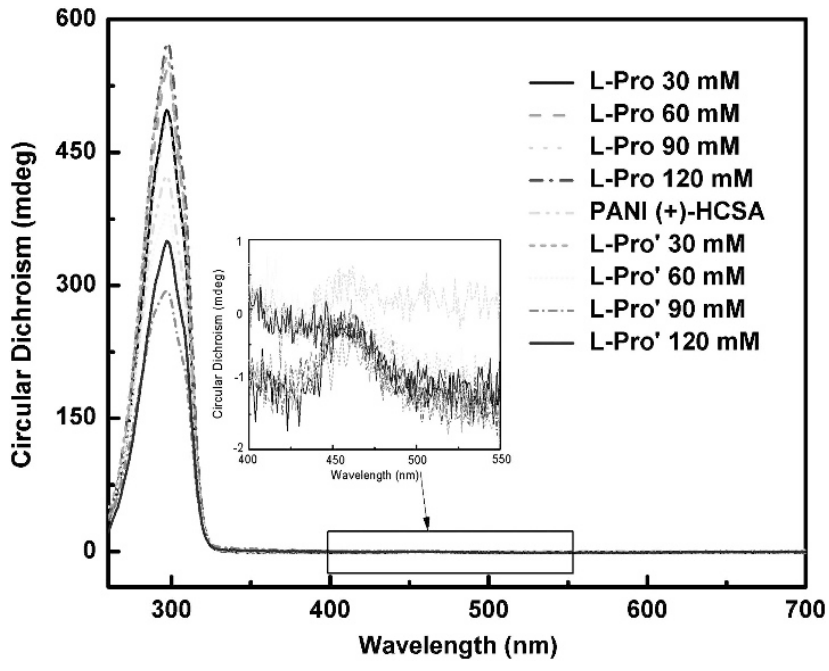

Figure 11 CD spectra of PANI(+)-HCSA in 30-120 mm of L-Pro along with enantiomeric PANI(+)-HCSA in DMF. (L-Pro spectra were taken within 30 min of doping, while L-Pro' were taken after $24 \mathrm{~h}$ of doping). A full color version of this figure is available at Polymer Journal online.

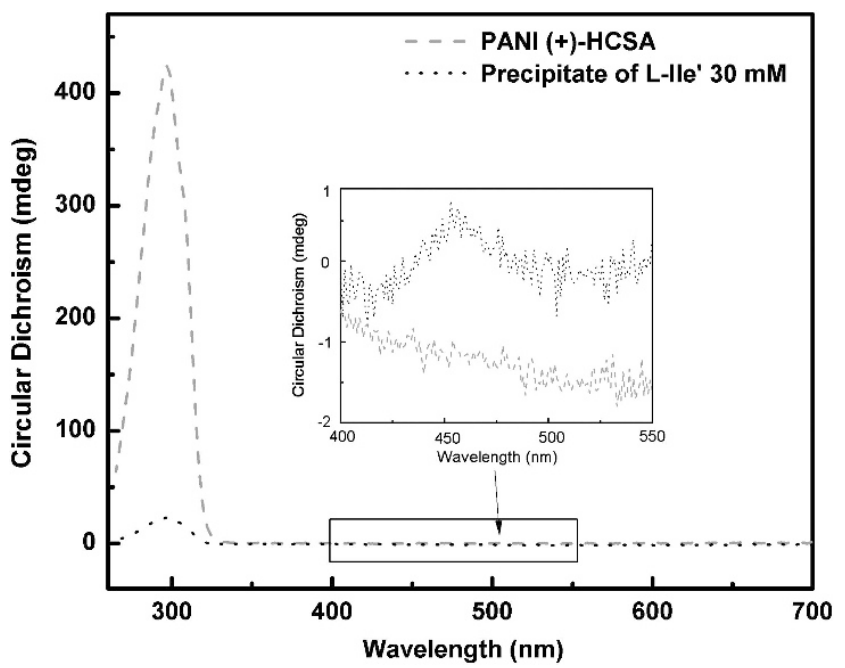

Figure $12 \mathrm{CD}$ spectra for PANI(+)-HCSA with $30 \mathrm{~mm}$ L-Ile in solid and solution phase along with enantiomeric PANI(+)-HCSA in DMF. A full color version of this figure is available at Polymer Journal online.

higher, whereas in the other concentrations these band intensities were found to be lower than that of the PANI $(+)$-HCSA solution. This result shows that there is a decrease in the extent of hydrogen bonding resulting in a decrease in band intensity with increased time.

The addition of different amino acids as precursors prior to the chiral HCSA acid doping of PANI had severe effect on the helicity. The UV-Visible and CD spectra of the amino acid doped helical PANI reveals that the nature and degree of band shifting depends on the amino acids used. It can therefore be concluded from the spectra that PANI (+)-HCSA shows bands at 295 and $455 \mathrm{~nm}$. In the case of L-Pro, L-Lys and L-Arg, the CD spectra show band shifts. The L-Lys shows the helicity band at $385 \mathrm{~nm}$, whereas the L-Pro shows a red shift (ca. $460 \mathrm{~nm}$ ). The L-Arg shows three bands corresponding to the UV-Visible spectra, in which the bands at 365 and $460 \mathrm{~nm}$ represent 
the compactness and coiling of the PANI chain; however, the band at $365 \mathrm{~nm}$ is not very prominent. The red shift suggests an increase in the conjugation length of the PANI chain. ${ }^{14}$ These band shifts help to discriminate between different amino acids.

After 15 days, the color of the PANI $(+)$-HCSA solutions changed from green to blue and their UV-Visible spectra were also found to be different from that of the EB form of PANI in DMF. As expected, the corresponding $\mathrm{CD}$ spectra exhibited no measurable optical activity and were consistent with complete de-doping of ES to EB. Further, on doping the solution, we obtained a precipitate which showed a remarkable optical activity with its $\mathrm{CD}$ spectra. Thus, we can infer that the optical activity of the solid form was retained even after the natural de-doping of ES to EB. This retention is indicated in Figure 12, which shows the CD spectra with a less intense peak for the retention of the optical activity in the solid phase, as obtained after de-doping of the solution.

\section{CONCLUSIONS}

The chiroptical properties of PANI $(+)$-HCSA are sensitive to the presence of chiral amino acids as precursors. There is a slight decrease in the optical activity of chiral PANI chains when they are converted back into the EB form. Thus, we conclude that the main factors influencing the chirality and stability of $\mathrm{PANI}(+)$-HCSA are stereochemistry of the amino acids, concentration of amino acids, time period for the doping, hydrogen bonding, chain length of the amino acids and the moieties present on the amino acids. Chiral discrimination between different amino acids is reported on the basis of $\mathrm{CD}$ band shifts. With control of these factors, a tailor-made helical conducting polymer can be designed which may be suitable for various technological applications.

\section{ACKNOWLEDGEMENTS}

We are thankful to Professor PB Sharma, Vice-Chancellor, Delhi Technological University (formerly Delhi College of Engineering), Delhi for encouragement and support. We are also thankful to DST, New Delhi for a financial grant under the DST-JSPS collaborative research program. Financial support to one of the authors, Sudha, is also acknowledged.
1 Kumar, D. \& Sharma, R. C. Advances in conductive polymers. Eur. Polym. J. 34, 1053-1060 (1998).

2 Peeters, E., Christiaans, M. P. T., Janssen, R. A. J., Schoo, H. F. M., Dekkers, H. P. J. M. \& Meijer, E. W. Circularly-polarized electroluminescence from a polymer light-emitting diode. J. Am. Chem. Soc. 119, 9909-9910 (1997).

$3 \mathrm{Pu}, \mathrm{L}$. Novel chiral conjugated macromolecules for potential electrical and optical applications. Macromol. Rapid Commun. 21, 795-809 (2000).

4 Fiesel, R., Halkyard, C. E., Rampey, M. E., Kloppenburg, L., Studer-Marinz, S. L., Scherf, U. \& Bunz, U. H. F. Aggregation and chiroptical behavior of a high molecular weight chirally substituted dialkylpoly(p-phenyleneethynylene). Macromol. Rapid Commun. 20, 107-111 (1999).

5 Moutet, J. C., Saint-Aman, E., Tan-Van, F., Angibeaud, P. \& Utille, J. P. Poly(glucosepyrrole) modified electrodes: a novel chiral electrode for enantioselective recognition. Adv. Mater. 4, 511-521 (1992).

6 MacDiarmid, A. G. \& Kaner, R. B. in Handbook of Conducting Polymers (ed. Skotheim, T.A.) 718 (Skothim Marcel Dekker, Inc, New York, 1986).

7 Gustafsson, G., Cao, Y., Treacy, G. M., Colaneri, N. \& Heeger, A. J. High performance photonic devices made with semiconducting polymers. Nature 357, 477-4779 (1992).

8 Yashima, E., Maeda, K., lida, H., Furusho, Y. \& Nagai, K. Helical polymers-synthesis, structures and functions. Chem. Rev. 109, 6102-6211 (2009).

9 Zhang, X. \& Song, W. Potential controlled electrochemical assembly of chiral polyaniline with enhanced stereochemical selectivity. Polymer 48, 5473-5479 (2007).

10 Pornputtkul, Y., Kane-Maguire, L. A. P. \& Wallace, G. G. Influence of electrochemical polymerization temperature on the chiroptical properties of $(+)$-Camphorsulfonic aciddoped polyaniline. Macromolecules 39, 5604-5610 (2006).

11 Yan, Y., Yu, Z., Huang, Y., Yuan, W. \& Wei, Z. Helical polyaniline nanofibers induced by chiral dopants by a polymerization process. Adv. Mater. 19, 3353-3357 (2007).

12 Yuan, G. L. \& Kuramoto, N. Helical polyaniline induced by specific interaction with biomolecules in neutral solution. Polymer 44, 5501-5504 (2003).

13 Ashraf, S. A., Kane-Maguire, L. A. P., Majidi, M. R., Pyne, S. G. \& Wallace, G. G. Influence of the chiral dopant anion on the generation of induced optical activity in polyanilines. Polymer 38, 2627-2631 (1997).

14 Egan, V., Bernstein, R., Hohmann, L., Tran, T. \& Kaner, R. B. Influence of water on the chirality of camphorsulfonic acid-doped polyaniline. Chem. Commun. 37, 801-802 (2001).

15 Boonchu, C., Kane-Maguire, L. A. P. \& Wallace, G. G. The effect of added water on the conformation of optically active polyaniline in organic solvents. Synth. Met. 135-136, 241-242 (2003).

16 Mire, C. A., Kane-Maguire, L. A. P. \& Wallace, G. G. Influence of added hydrogen bonding agents on the chiroptical properties of chiral polyaniline. Synth. Met. 159, 715-717 (2009).

17 Draman, S. F. S., Daik, R. \& Ahmad, M. Synthesis and studies on fluorescence spectroscopy of CSA-doped polyaniline solution in DMF when exposed to oxygen gas. Malay Polym. J. 4, 7-18 (2009).

18 Kane-Maguire, L. A. P., MacDiarmid, A. G., Norris, I. D., Wallace, G. G. \& Zheng, W. Facile preparation of optically active polyanilines via the in situ chemical oxidative polymerisation of aniline. Synth. Met. 106, 171-176 (1999).

Supplementary Information accompanies the paper on Polymer Journal website (http://www.nature.com/pj) 